\title{
Optical properties of potential condensates in exoplanetary atmospheres
}

\author{
Daniel Kitzmann ${ }^{\star}$ and Kevin Heng ${ }^{\star}$ \\ Center for Space and Habitability, Universität Bern, Gesellschaftsstr. 6, CH-3012 Bern, Switzerland
}

Accepted 2017 December 1. Received 2017 November 30; in original form 2017 October 13

\begin{abstract}
The prevalence of clouds in currently observable exoplanetary atmospheres motivates the compilation and calculation of their optical properties. First, we present a new open-source Mie scattering code known as LX-MIE, which is able to consider large-size parameters $\left(\sim 10^{7}\right)$ using a single computational treatment. We validate LX-MIE against the classical MIEvo code as well as previous studies. Secondly, we embark on an expanded survey of the published literature for both the real and imaginary components of the refractive indices of 32 condensate species. As much as possible, we rely on experimental measurements of the refractive indices and resort to obtaining the real from the imaginary component (or vice versa), via the KramersKronig relation, only in the absence of data. We use these refractive indices as input for LX-MIE to compute the absorption, scattering and extinction efficiencies of all 32 condensate species. Finally, we use a three-parameter function to provide convenient fits to the shape of the extinction efficiency curve. We show that the errors associated with these simple fits in the Wide Field Camera 3 (WFC3), $J, H$, and $K$ wavebands are $\sim 10$ per cent. These fits allow for the extinction cross-section or opacity of the condensate species to be easily included in retrieval analyses of transmission spectra. We discuss prospects for future experimental work. The compilation of the optical constants and LX-MIE is publicly available as part of the open-source Exoclime Simulation Platform (http://www.exoclime.org).
\end{abstract}

Key words: planets and satellites: atmospheres - planets and satellites: gaseous planetsbrown dwarfs.

\section{INTRODUCTION}

Clouds and hazes are expected to be commonplace (Marley et al. 2013) and have been observationally established to be prevalent in currently observable exoplanetary atmospheres (Pont et al. 2008; Sing et al. 2016), with the degree of cloudiness being tentatively correlated with gravity and temperature (Heng 2016; Stevenson et al. 2016). These findings motivate the inclusion of the effects of these clouds and hazes into retrieval analyses of the spectra of exoplanetary atmospheres. Cloud and haze formation from first principles is a challenging topic (e.g. Helling, Woitke \& Thi 2008), but once they are formed their effects on the spectrum is a well-understood phenomenon as long as their optical properties are known, because the absorption, scattering, and extinction efficiencies may be computed using Mie theory (e.g. van de Hulst 1957; Pierrehumbert 2010), as we aim to do in the current study. Effectively, a problem involving particle kinetics embedded in a magnetized fluid reduces to an optics problem. This reasoning motivates the compilation of a library of refractive indices and the calculation of the corresponding absorption, scattering, and extinction efficiencies.

^E-mail: daniel.kitzmann@csh.unibe.ch (DK); kevin.heng@csh.unibe.ch $(\mathrm{KH})$
In this study, we survey the published literature to obtain the measured real and imaginary components of the refractive indices of 32 condensate species. This expands upon the 20 species considered by Wakeford \& Sing (2015). A subset of 12 species from the compilation of Wakeford \& Sing (2015) has recently been used by Pinhas \& Madhusudhan (2017) to obtain extinction cross-sections of potential condensates and their spectral signatures. In this work, we also revise some of their used optical constants by using alternative data sources (e.g. for $\mathrm{TiO}_{2}$ ). We especially also include condensates that are expected to form in carbon-rich environments, such as, for example, TiC.

Additionally, we try to cover the entire wavelength range from the ultraviolet to the far-infrared for each species, which makes the compilation suitable for atmospheric modelling and simulating spectra. Heating from the star extends into the ultraviolet (e.g. the Ly $\alpha$ line at $0.12 \mu \mathrm{m}$ ), and for the purpose of radiative transfer it is essential to compute the extinction of starlight across depth or pressure. The presence of condensates could alter this extinction profile, which is why it is necessary to have extinction efficiencies to $\sim 0.1 \mu \mathrm{m}$. On the other hand, exoplanetary atmospheres emit thermally at peak wavelengths $\sim 1 \mu \mathrm{m}$ $(T / 1000 \mathrm{~K})$ and also longward of these wavelengths. To enforce radiative equilibrium requires that one is able to integrate the flux over all wavelengths, down into the long-wavelength tail of the thermal distribution. The presence of condensates could alter the 
profile of the flux across wavelength, and it is necessary to be able to compute extinction efficiencies to wavelengths as red as $\sim 10-100 \mu \mathrm{m}$.

We use these refractive indices as input for a newly constructed Mie scattering code, LX-MIE, which we use to compute the absorption, scattering, and extinction efficiencies of these 32 condensate species. Based on the reasoning that the extinction efficiency curve has a somewhat general shape (e.g. Pierrehumbert 2010), we fit a simple three-parameter function to it and quantify the errors associated with the fit in the WFC3, $J, H$, and $K$ wavebands for all 32 species. These empirical fits allow for the cross-section or opacity of these 32 condensate species to be easily included in retrieval analysis of transmission spectra, as was done by Lee, Heng \& Irwin (2013), Lavie et al. (2017), Oreshenko et al. (2017), and Tsiaras et al. (2017).

In Section 2, we first briefly review Mie theory as a prelude to describing the improved computational method for our LX-MIE code in Section 3. Our compilation and treatment of optical constants are described in Section 4. We present the three-parameter fits of the extinction efficiencies in Section 5, and finally discuss prospects for future work in Section 6.

\section{REVIEW OF MIE THEORY}

To calculate the optical properties of potential condensates, we use Mie theory (Mie 1908), assuming a spherical particle shape. For these calculations, we constructed a new Mie scattering code that is especially designed to handle situations with large-size parameters. Publicly available Mie codes, such as the well-known MIEvo code by Wiscombe (1980, 1979), are validated only for size parameters of the order of $10^{4}$. This, for example, is insufficient to treat particles with radii of $1000 \mu \mathrm{m}$ at ultraviolet wavelengths. Such large particles are, for example, found in the atmosphere of Earth in the form of ice crystals (Schmitt et al. 2016). Large particle sizes can usually be expected if clouds form from the major atmospheric constituent. If such an atmosphere becomes supersaturated, cloud particles can grow quickly due to the huge amount of available, condensible material. For example, Colaprete \& Toon (2003) estimated that the mean size of $\mathrm{CO}_{2}$ ice particles in a carbon dioxide-dominated atmosphere of early Mars would be $1000 \mu \mathrm{m}$. Other astrophysical environments where large dust particles are known to exist are protoplanetary discs. The dust grains in these discs can easily grow to sizes in the centimetre range (Birnstiel, Klahr \& Ercolano 2012; Mordasini 2014).

While geometric optics approximations can be used to determine the optical properties of large particles, it would be preferable to have a Mie code, able to describe the entire wavelength range of interest without needing to switch to different approximations at certain wavelengths and particle sizes. The Mie scattering code described in the following can easily handle size parameters of the order of $10^{7}$ and higher, including the calculation of the scattering and absorption efficiencies, asymmetry parameter, as well as the scattering phase function and its Legendre moments.

Condensates in astrophysical environments are probably not spherical but rather fractal-like structures. However, in situ measurements of the actual particle shapes in these environments are unavailable. Furthermore, the scattering properties of such non-spherical particles are extremely computationally demanding (Rother 2009). Thus, for simplicity, we employ the approximation of spherical particles in the following. Mie theory can also be used to approximate the optical properties of non-spherical or fractal particles. This was done, for example, for fractal haze particles in the atmosphere of the early Earth by Arney et al. (2016) or to describe non-spherical water ice cloud crystals in Earth-like atmospheres by Kitzmann et al. (2010).

With the assumption of spherical particles the cross-sections for a distinct particle radius $a$ and wavelength $\lambda$ are then given by (van de Hulst 1957; Bohren \& Huffman 1998)

$$
\begin{aligned}
C_{\mathrm{ext}}(a, \lambda)= & \frac{2 \pi a^{2}}{x^{2}} \sum_{n=1}^{\infty}(2 n+1) \operatorname{Re}\left(a_{n}(m(\lambda), x)+b_{n}(m(\lambda), x)\right) \\
C_{\mathrm{sca}}(a, \lambda)= & \frac{2 \pi a^{2}}{x^{2}} \sum_{n=1}^{\infty}(2 n+1) \\
& \times\left(\left|a_{n}(m(\lambda), x)\right|^{2}+\left|b_{n}(m(\lambda), x)\right|^{2}\right) \\
C_{\mathrm{abs}}(a, \lambda)= & C_{\mathrm{ext}}(a, \lambda)-C_{\mathrm{sca}}(a, \lambda)
\end{aligned}
$$

with the dimensionless size parameter

$x=\frac{2 \pi a}{\lambda}$.

The Mie coefficients $a_{n}(m(\lambda), x)$ and $b_{n}(m(\lambda), x)$ are defined with respect to the wavelength-dependent complex refractive index

$m(\lambda)=n(\lambda)-i k(\lambda)$

of the considered material. ${ }^{1}$ For a monodisperse population of condensates, it is convenient to specify the dimensionless efficiencies,

$Q=\frac{C}{\pi a^{2}}$,

instead of the corresponding cross-sections.

\subsection{Scattering phase function}

To quantify the role of scattered radiation for, for example, the scattering greenhouse effect of cloud particles in extrasolar planetary atmospheres, the corresponding angular distribution of the scattered radiation has to be specified by a scattering phase function $p(a, \alpha)$, which describes the probability of a photon to be scattered with a scattering angle $\alpha$. The Mie intensity functions $i_{1}(a, \alpha)$ and $i_{2}(a, \alpha)$

$$
\begin{aligned}
& i_{1}(a, \alpha)=\left|\sum_{n=1}^{\infty} \frac{2 n+1}{n(n+1)}\left[a_{n}(m, x) \pi_{n}(\alpha)+b_{n}(m, x) \tau_{n}(\alpha)\right]\right|^{2}, \\
& i_{2}(a, \alpha)=\left|\sum_{n=1}^{\infty} \frac{2 n+1}{n(n+1)}\left[a_{n}(m, x) \tau_{n}(\alpha)+b_{n}(m, x) \pi_{n}(\alpha)\right]\right|^{2}
\end{aligned}
$$

are used for the description of $p(a, \alpha)$. The angular eigenfunctions $\pi_{n}(a, \alpha)$ and $\tau_{n}(a, \alpha)$ are defined as

$\pi_{n}(a, \alpha)=\frac{P_{n}^{1}(\cos \alpha)}{\sin \alpha} \quad$ and $\quad \tau_{n}(a, \alpha)=\frac{\mathrm{d} P_{n}^{1}(\cos \alpha)}{\mathrm{d} \alpha}$,

where $P_{n}^{j}$ are the associated Legendre polynomials. The scattering phase function $p(a, \alpha)$ is given in terms of these intensities by ${ }^{2}$

$p(a, \alpha)=\frac{\lambda^{2}}{2 \pi} \frac{i_{1}(a, \alpha)+i_{2}(a, \alpha)}{C_{\mathrm{sca}}(a)}$.

\footnotetext{
${ }^{1}$ In the following, we are dropping the $\lambda$ for the simplification of notation.

${ }^{2}$ We note that the phase function defined here corresponds to Wiscombe (1980) but it differs from that given in van de Hulst (1957) or Bohren \& Huffman (1998) by a factor of $4 \pi$.
} 
It can also be represented as a series of Legendre polynomials (Chandrasekhar 1960)

$p(a, \alpha)=\sum_{n=0}^{\infty}(2 n+1) P_{n}^{0}(\cos \alpha) \chi_{n}(a)$,

with

$\chi_{n}(a)=\frac{1}{2} \int_{-1}^{1} p(a, \alpha) P_{n}^{0}(\cos \alpha) \mathrm{d} \cos \alpha$.

In practise, the phase function expansion series is truncated at $n=N_{\max }$ where the $\chi_{N_{\max }}(a)$ becomes numerically zero.

The asymmetry parameter $g(a)$ is another important parameter to characterize the scattering properties of cloud particles. It is defined as the mean cosine of the scattering angle

$g(a)=\langle\cos \alpha\rangle=\frac{1}{2} \int_{0}^{\pi} p(\alpha) \cos (\alpha) \sin (\alpha) \mathrm{d} \alpha$.

If more light is scattered in forward direction $\left(\alpha>90^{\circ}\right), g$ is positive, while $g<0$ is obtained for dominant scattering in the backward direction $\left(\alpha>90^{\circ}\right)$. For $g=0$, the phase function is symmetric around $\alpha=90^{\circ}$ (but not necessarily isotropic). Note, that $g$ exactly corresponds to the $\chi_{1}$ moment of the Legendre series expansion (cf. equation 9).

\section{AN IMPROVED MIE CODE: LX-MIE}

The optical properties of spherical particles can be determined from equation (1). The most difficult task is thereby the calculation of the Mie coefficients $a_{n}$ and $b_{n}$, given by

$a_{n}(m, x)=\frac{\psi_{n}(x) \psi_{n}^{\prime}(m x)-m \psi_{n}^{\prime}(x) \psi_{n}(m x)}{\zeta_{n}(x) \psi_{n}^{\prime}(m x)-m \zeta_{n}^{\prime}(x) \psi_{n}(m x)}$,

$b_{n}(m, x)=\frac{m \psi_{n}(x) \psi_{n}^{\prime}(m x)-\psi_{n}^{\prime}(x) \psi_{n}(m x)}{m \zeta_{n}(x) \psi_{n}^{\prime}(m x)-\zeta_{n}^{\prime}(x) \psi_{n}(m x)}$

with the Riccati-Bessel functions $\zeta_{n}, \psi_{n}$ and their corresponding derivatives (Bohren \& Huffman 1998). In the following, we first briefly summarize the standard approach for the calculation of $a_{n}$ and $b_{n}$. However, under certain conditions particles might reach very large size parameters of the order of $10^{5}$, which cannot be handled by the standard Mie calculation procedure. Consequently, we developed a new modified Mie-program code LX-MIE, especially suited for large size parameters $x$. The improvements we introduced in these Mie calculations are discussed in Section 3.2.

\subsection{Standard procedure for Mie calculations}

The calculation of the Mie coefficients present several computational challenges. The most important one is the calculation of $\psi_{n}^{\prime}(m x)$ and $\psi_{n}(m x)$, which can be solved by introducing the logarithmic derivative $A_{n}(x)$ (Infeld 1947)

$A_{n}(m x)=\frac{\mathrm{d}\left(\log \psi_{n}(m x)\right)}{\mathrm{d}(m x)}=\frac{\psi_{n}^{\prime}(m x)}{\psi_{n}(m x)}=-\frac{n}{m x}+\frac{J_{n-1 / 2}(m x)}{J_{n+1 / 2}(m x)}$,

where $J_{n \pm 1 / 2}$ are the Bessel functions of the first kind. Using the logarithmic derivative, the Mie coefficients can be re-written in the following form:

$a_{n}(m, x)=\frac{\left(A_{n}(m x) / m+n / x\right) \psi_{n}(x)-\psi_{n-1}(x)}{\left(A_{n}(m x) / m+n / x\right) \zeta_{n}(x)-\zeta_{n-1}(x)}$,
$b_{n}(m, x)=\frac{\left(m A_{n}(m x)+n / x\right) \psi_{n}(x)-\psi_{n-1}(x)}{\left(m A_{n}(m x)+n / x\right) \zeta_{n}(x)-\zeta_{n-1}(x)}$.
The logarithmic derivative $A_{n}(m x)$ can then be obtained by an upward or a downward recursion. The upward recursion is only stable for special cases [e.g. if $\operatorname{Im}(m)$ is small], while the downward recursion

$A_{n-1}(m x)=\frac{n}{m x}-\frac{1}{\frac{n}{m x}+A_{n}(m x)}$

is always stable (Wiscombe 1979). For a downward recursion, however, the initial value $A_{N}(m x)$ must be known a priori, where $n=N$ is the last term to be taken into account in the (in principle infinite) Mie series. Based on numerical studies with size parameters of up to 20000 , Wiscombe (1980) suggested the following values:

$N=\tilde{N}(x)=\left\{\begin{array}{ll}x+4 x^{1 / 3}+1 & 0.02 \leq x \leq 8 \\ x+4.05 x^{1 / 3}+2 & 8<x<4200 \\ x+4 x^{1 / 3}+2 & 4200 \leq x \leq 20000\end{array}\right.$.

This commonly used criterion for the value of $N=\tilde{N}(x)$ from Wiscombe (1980) was derived empirically for $x \leq 20000$, but lacks a clear mathematical justification. The corresponding initial value $A_{N}(m x)$ for the recurrence relation is usually obtained by the continuous fraction method introduced by Lentz (1976). ${ }^{3}$ The RiccatiBessel functions $\zeta_{n}(x)$ and $\psi_{n}(x)=\operatorname{Re} \zeta_{n}(x)$ are calculated by an upward recursion (Abramowitz \& Stegun 1972), i.e.

$\zeta_{n+1}(x)=\frac{2 n+1}{x} \zeta_{n}(x)-\zeta_{n-1}(x)$

This recursion, however, is not always stable, especially if $n \gg$ $x$ numerical errors start to dominate the calculations making the upward recursion unstable.

\subsection{Improved Mie calculations for large-size parameters}

To perform Mie calculations for large size parameters, the method to calculate the Riccati-Bessel functions in the Mie coefficients has to be improved. Therefore, following Shen \& Cai (2005), we write the Mie coefficients $a_{n}$ and $b_{n}$ in the form

$$
\begin{aligned}
& a_{n}(m, x)=B_{n}(x) \frac{A_{n}(m x) / m-A_{n}(x)}{A_{n}(m x) / m-C_{n}(x)}, \\
& b_{n}(m, x)=B_{n}(x) \frac{A_{n}(m x) m-A_{n}(x)}{A_{n}(m x) m-C_{n}(x)}
\end{aligned}
$$

with

$$
\begin{aligned}
& B_{n}(x)=\frac{\psi_{n}(x)}{\zeta_{n}(x)}, \\
& C_{n}(x)=\frac{\zeta_{n}^{\prime}(x)}{\zeta_{n}(x)} .
\end{aligned}
$$

In this formulation, the Mie coefficients are only functions of ratios of Riccati-Bessel functions and their derivatives. Thus, the direct calculation of single Riccati-Bessel functions is completely avoided. The computation of these ratios is numerically much more stable than that of single Riccati-Bessel functions, which results in higher accuracy and computational stability for large $x$ and $n$. Therefore, large size parameters of, for example, $x=10^{6}$ can be used without numerical problems in our Mie calculations.

\footnotetext{
${ }^{3}$ We note that in principle $A_{n}(m x)$ can be calculated with the continuous fractions from Lentz (1976) for any value of $n$, but the recurrence relation equation (14) is computationally much faster.
} 
The ratios $B_{n}(x)$ and $C_{n}(x)$ also obey recurrence relations, namely

$$
\begin{aligned}
& B_{n}(x)=B_{n-1}(x) \frac{C_{n}(x)+n / x}{A_{n}(x)+n / x}, \\
& C_{n}(x)=-\frac{n}{x}+\frac{1}{\frac{n}{x}-C_{n-1}(x)} .
\end{aligned}
$$

Both ratios are calculated here via upward recursions, starting with

$$
\begin{aligned}
& B_{1}(x)=\left(1+\mathrm{i} \frac{\cos x+x \sin x}{\sin x-x \cos x}\right)^{-1} \text { and } \\
& C_{0}(x)=-\mathrm{i} .
\end{aligned}
$$

The coefficients $A_{n}(m x)$ and $A_{n}(x)$ are again calculated via the downward recurrence equation (14), and its initial values $A_{N}(m x)$ and $A_{N}(x)$ are obtained from the continuous fraction method after Lentz (1976).

To obtain a non-empirical criterion for the value of $N=N(x)$ in the case of large $x$, we adopt the method published by Cachorro \& Salcedo (1991). They proved mathematically that the convergence properties of the Mie series depend almost completely on the ratios $B_{n}(x)$ in equation (17). Based on their findings they concluded that the truncation criterion for the series (i.e. the value of $N$ ) as a function of the desired accuracy $\epsilon$ is given by

$\left|\operatorname{Im} \zeta_{N}(x)\right| \geq \sqrt{\epsilon^{-1}}$,

considering the fact $\operatorname{Re} \zeta_{n}(x)=\psi_{n}(x)$ is negligible compared to $\operatorname{Im} \zeta_{n}(x)$ for large values of $n$. For their general relation of the truncation criterion

$N=\tilde{N}(x)=x+c \times x^{1 / 3}$,

they derived the constants $c$ for several values of $\epsilon$. For our Mie calculations, we adopt their value of $c=4.3$, which corresponds to $\epsilon=10^{-8}$.

However, both the criteria of Wiscombe (1980) and of Cachorro \& Salcedo (1991) are only functions of the size parameter $x$ and do not depend on the refractive index $m$. As discussed by Cachorro \& Salcedo (1991), the conclusion of $\tilde{N}$ being only a function of $x$ is not universally true. In extremely rare cases resonant terms can exist in the Mie series (depending on $m$ ), which invalidates the assumption that $\tilde{N}$ is a function of $x$ only. In order for these resonance effects to appear in the Mie series, $x$ and $m$ would have to be fine tuned with very high precision (Cachorro \& Salcedo 1991). Therefore, it is unlikely to encounter such resonances in practical Mie calculations by coincidence.

With given Mie coefficients, the remaining calculations are straightforward. For the Mie intensities (equation 5), the angular eigenfunctions $\pi_{n}(a, \alpha)$ and $\tau_{n}(a, \alpha)$ can also be written as recurrence relations, which are calculated via upward recursion (see Wiscombe 1979 for details). To calculate the moments of the Legendre series expansion of the scattering phase function (equation 9), the procedure from Dave (1970) can be used. The newly developed Mie scattering code LX-MIE has been applied to various test examples for validation (see Appendix A).

\section{OPTICAL CONSTANTS}

In this section, we describe a new collection of optical constants, the real part $n$ and the imaginary part $k$ of the refractive index, for a broad set of potential condensates expected in extrasolar gas planets.

Previously, a set of optical constants for 20 species was published by Wakeford \& Sing (2015). We here extend and revise their published optical constants by including more dust species and exploring other data sources to obtain refractive indices over a broad wavelength range from the ultraviolet to the far-infrared. The only two species that have the same optical constants in Wakeford \& Sing (2015) and this publication are $\mathrm{KCl}$ and $\mathrm{NaCl}$. We note that the data set for the refractive index of $\mathrm{TiO}_{2}$ (Kangarloo \& Rafizadeh 2010a,b) used by Wakeford \& Sing (2015) has been retracted by the corresponding journal and, thus, requires revision. We especially focus on amorphous condensates, which are probably the more common form of dust particles in astrophysical environments. In addition to Wakeford \& Sing (2015), we also take anisotropic crystals and temperature-dependent refractive indices into account, if the corresponding data is available.

Our list includes several common silicates, oxides, sulfides, chlorides, but also carbon bearing condensates. We also include some solid solutions of the olivine and pyroxene groups. Most of the optical constants are given from the ultraviolet to the far-infrared range of wavelengths, which makes them suitable to be included in atmospheric models or to study their impact on spectra. The optical constants have been obtained from several sources and are listed in Table 1 . The plots of the $n$ and $k$ values as a function of wavelength for all considered condensates are shown in Fig. 1. For enstatite and quartz, we respectively include two different data sets. In case of $\mathrm{MgSiO}_{3}$, these are two different amorphous states, and for $\mathrm{SiO}_{2}$, we include its $\alpha$-crystal as well as amorphous form.

In theory, $n$ and $k$ are not independent but as the real and imaginary part of a complex number must follow the Kramers-Kronig relations (Kronig 1926; Kramers 1927)

$n(\omega)-1=\frac{2}{\pi} P \int_{0}^{\infty} \frac{\omega^{\prime} k\left(\omega^{\prime}\right)}{\omega^{\prime 2}-\omega^{2}} \mathrm{~d} \omega^{\prime}$
$k(\omega)=-\frac{2 \omega}{\pi} P \int_{0}^{\infty} \frac{n\left(\omega^{\prime}\right)-1}{\omega^{\prime 2}-\omega^{2}} \mathrm{~d} \omega^{\prime}$,

where $\omega=2 \pi c / \lambda$ is the angular frequency and $P$ denotes the principal value of the singular integral. Theoretically, all $n$ and $k$ values shown in Fig. 1 must satisfy these two relations. In practice, however, this is not always the case. Since most of the data is compiled from different sources, with diverse experimental setups, measurement errors, etc., it is usually impossible to satisfy the KramersKronig relations over the entire wavelength range. While it would be possible to iterate $n$ and $k$ via the Kramers-Kronig relations to obtain a consistent set of optical constants, the usefulness of this approach is limited by the fact that the resulting refractive index would not be consistent anymore with the measured experimental data. Since we do not wish to judge each experimentally derived refractive index on the basis of their experimental setup or measurement accuracy, we choose not to perform an iteration here.

Furthermore, the calculation of $k$ in the large transparency windows, where it attains values of smaller than say $10^{-5}$ would mean that $n$ would have to be measured with a comparable accuracy in order to produce reliable $k$-values via the Kramers-Kronig relations. Experimental measurement errors, however, are well above this required accuracy.

Gaps in the experimental data are closed by extrapolation of either $n$ or $k$ and then using the Kramers-Kronig relations to obtain the other, missing part of the refractive index over the wavelength range of the data gap. For this procedure, we employ the singlysubtractive Kramers-Kronig relations that use experimental data at other wavelengths to constrain the missing coefficients in the data gap (Lucarini et al. 2005).

In case of $\mathrm{MnS}$, no infrared data was available, which includes, in particular, the sulphur feature. Here, we follow the approach of 
Table 1. Collection of optical constants for a set of potential condensates in atmospheres of extrasolar planets and brown dwarfs. The Comments column lists additional information on temperature-dependent data (if available), and amorphous or anisotropic materials. If no temperature information is given, measurements where either performed at room temperature or no information is provided in the corresponding publications.

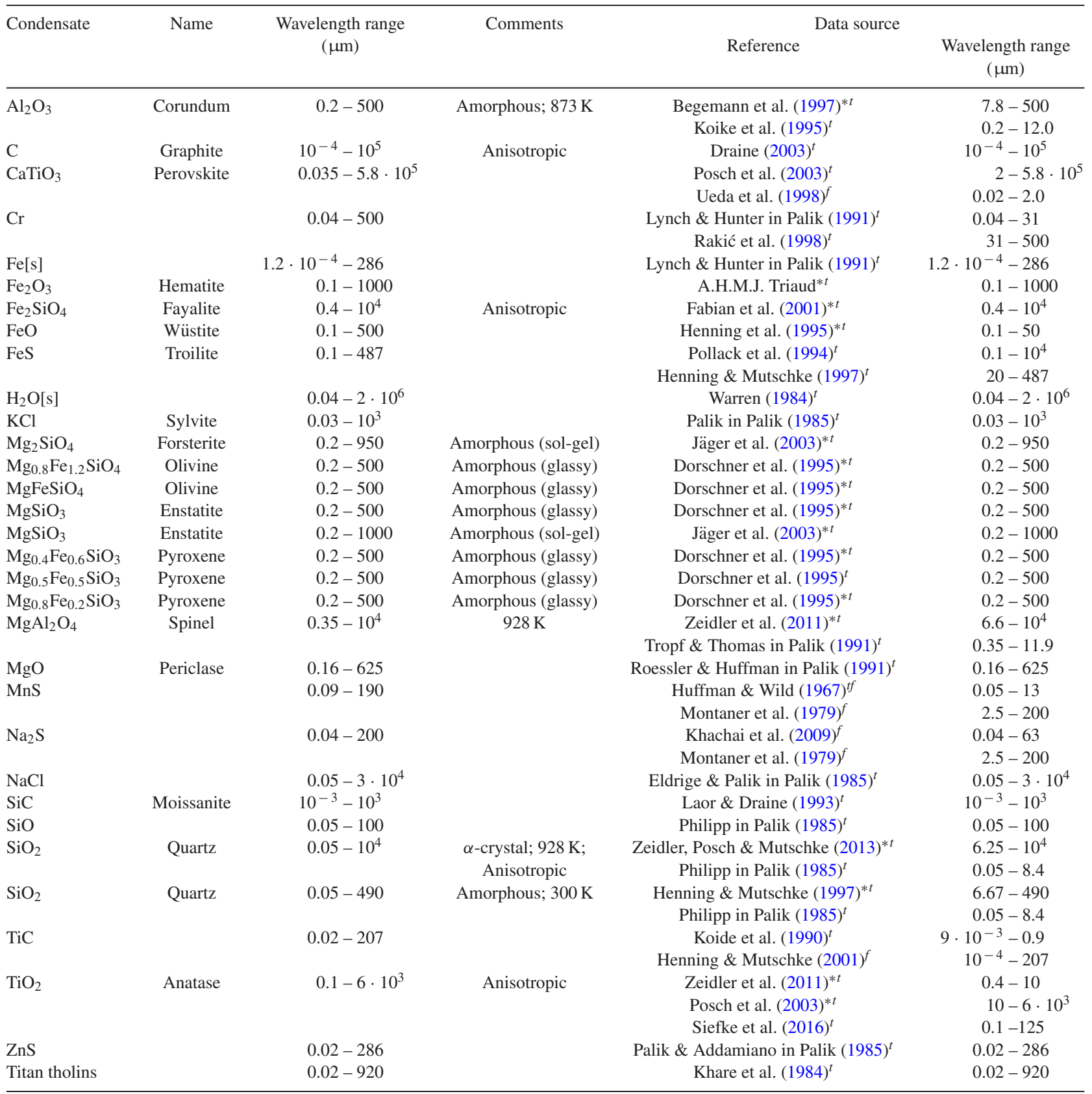

Notes. ${ }^{*}$ Optical constants from the Database of Optical Constants for Cosmic Dust, Laboratory Astrophysics Group of the AIU Jena (http://www.astro.uni-jena.de/Laboratory/OCDB/index.html).

${ }^{t}$ Data from a printed table or in digital format.

${ }^{f}$ Data available as figure.

Morley et al. (2012) and use extrapolations based on the other two sulphur-bearing species $\mathrm{Na}_{2} \mathrm{~S}$ and $\mathrm{ZnS}$ to reconstruct the missing part. For troilite, we combine the two data sets of Pollack et al. (1994) and Henning \& Mutschke (1997). The compilation of Pollack et al. (1994) used a few experimental data points in combination with extrapolations to account for the missing data. Especially in the infrared region, this set is not consistent with the measured data provided by Henning \& Mutschke (1997). We therefore replace the IR data with the latter one and use the Kramers-Kronig relations to consistently combine the IR part with the measured shortwave data provided in Pollack et al. (1994). This leads to a shift of the strong iron feature near $3 \mu \mathrm{m}$ to slightly larger wavelengths compared to the original compilation provided by Pollack et al. (1994).

Some condensates, such as graphite, are anisotropic, i.e. their optical properties depend on the direction of the incident light relative to the crystals' principal axes. In case directional-dependent data was available for these condensates, we calculate a mean refractive index by weighting the dielectric functions in each direction by $1 / 3$ 


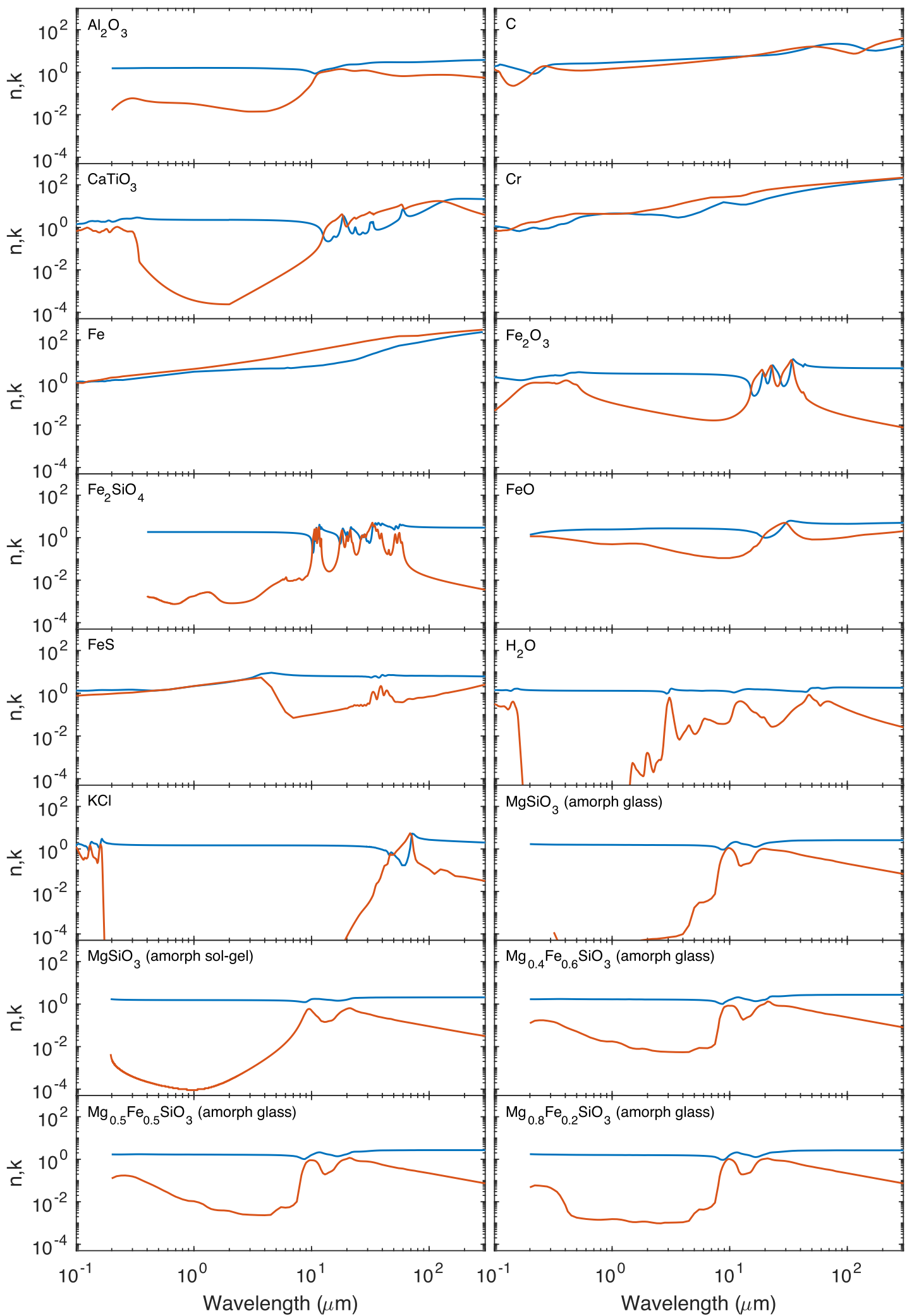

Figure 1. Optical constants of a set of potential condensates. The plots show the real ( $n$, blue) and imaginary $(k$, red) parts of the refractive index. The optical constants have been compiled from several different sources. Details on the compilation are given in Section 4 and Table 1. 

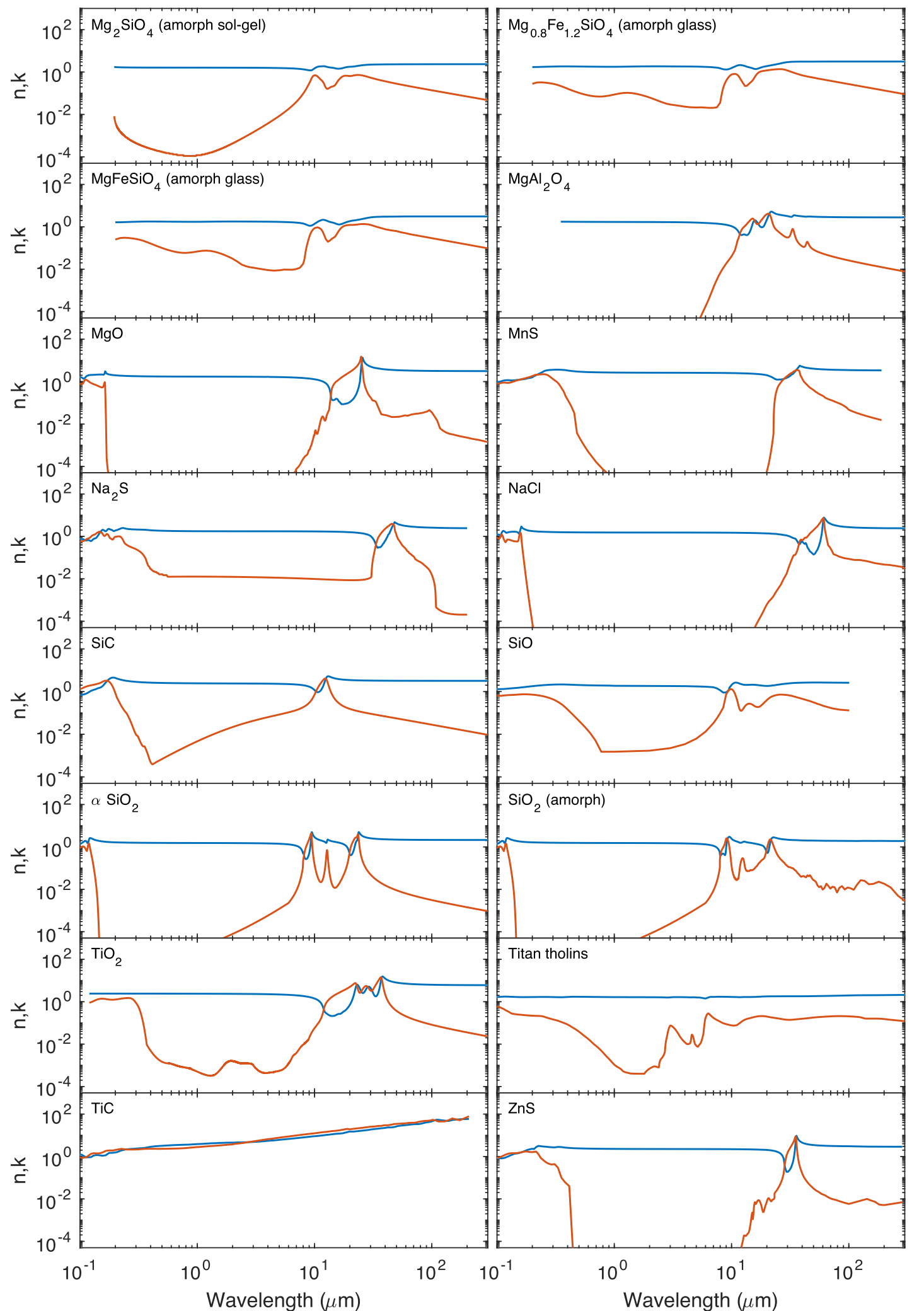

$\mathrm{SiO}_{2}$ (amorph)

Figure 1 - continued 
and converting them to the corresponding $n$ and $k$ values. In Table 1 , these materials are marked as anisotropic.

In case temperature-dependent data is available, we always choose a temperature close to the ones expected in atmospheres of brown dwarfs or giant exoplanet where the condensates are expected to form. In most cases, though, the optical constants have been measured at room temperature.

\section{ANALYTICAL FitS OF EXTINCTION EF F I CIENCIES}

In retrieval analyses of spectra of exoplanetary atmospheres, there is always a balance between the realism and sophistication of the model, the computational efficiency, and the quality and number of data points. Including a full-blown, first-principles cloud model in atmospheric retrievals of current spectra is currently unwarranted, given the large number of free parameters involved and the $\sim 10-100$ data points typically available for each object. Instead of including a formation model of clouds in the retrieval, several past efforts have focused on how the clouds would affect the spectrum if assumed to be present (Lee et al. 2013; Lavie et al. 2017; Oreshenko et al. 2017; Tsiaras et al. 2017). Specifically, these studies follow the reasoning laid out in, for example, Pierrehumbert (2010), that the curves of extinction efficiencies follow a roughly universal shape, somewhat insensitive to the composition of the condensate. (We will see later that this statement is only partially correct.) The efficiencies for small and large values of the size parameter $(x)$ converge to simpler, analytical expressions. In other words, our motivation is to provide a library of more accurate fits to augment the approach that is already being pursued in the published literature.

For large values of $x$, i.e. if the particle radius is large compared to the wavelength under consideration, Mie theory yields the largeparticle limit, which is given by the simple expressions

$$
\begin{aligned}
& \lim _{x \rightarrow \infty} Q_{\text {abs }}=1-Q_{\text {refl }}, \\
& \lim _{x \rightarrow \infty} Q_{\text {sca }}=1+Q_{\text {refl }}
\end{aligned}
$$

and

$\lim _{x \rightarrow \infty} Q_{\text {ext }}=2$.

The reflection efficiency $Q_{\text {refl }}$ can be determined by geometric optics calculations (see Bohren \& Huffman 1998 for details).

For particles much smaller than the considered wavelength, i.e. $x=2 \pi a / \lambda \ll 1$, Mie theory converges to the limit of Rayleigh scattering. The efficiencies are then given by

$$
\begin{aligned}
Q_{\mathrm{abs}} & =4 x \Im\left\{\frac{m(\lambda)^{2}-1}{m(\lambda)^{2}+2}\right\}\left[1-\frac{4 x^{3}}{3} \Im\left\{\frac{m(\lambda)^{2}-1}{m(\lambda)^{2}+2}\right\}^{2}\right], \\
Q_{\text {sca }} & =\frac{8}{3} x^{4} \Re\left\{\frac{m(\lambda)^{2}-1}{m(\lambda)^{2}+2}\right\}^{2},
\end{aligned}
$$

and

$$
Q_{\text {ext }}=Q_{\text {abs }}+Q_{\text {sca }} \text {. }
$$

An example of the extinction efficiencies of graphite for three different particle sizes is shown in Fig. 2. The figure illustrates nicely the two different limits of Mie theory just discussed. Note, however, that the curve for the particles with a radius of $0.1 \mu \mathrm{m}$ slightly deviates from the large particle limit due to a drop in the absorptivity of graphite at around a wavelength of $0.1 \mu \mathrm{m}$ (see Fig. 1). The

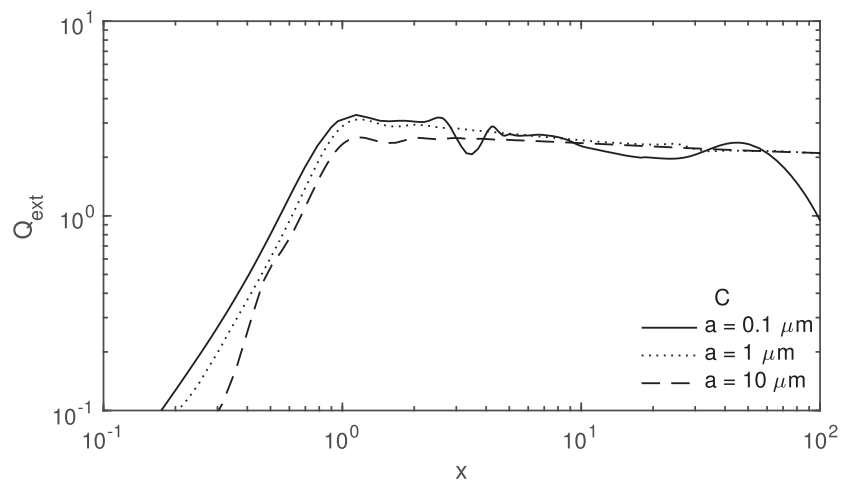

Figure 2. Extinction efficiency of graphite as a function of the size parameter $x$. Shown are the results for three different particle sizes: $0.1 \mu \mathrm{m}$ (solid), $1 \mu \mathrm{m}$ (dotted), and $10 \mu \mathrm{m}$ (dashed).

overall shape of the curve can, in principle, be represented by an almost constant value at large $x$, a slope for small size parameters, and a specific $x$-value where the $Q_{\text {ext }}$ peaks, connecting the two limits.

Given the general shape of the extinction efficiency curve, Lee et al. (2013) proposed a simple analytical fit to describe the overall behaviour of $Q_{\text {ext }}$ as a function of the size parameter $x$

$Q_{\mathrm{ext}}=\frac{5}{Q_{0} x^{-4}+x^{0.2}}$.

This fit is not expected to provide an accurate representation of the extinction efficiencies but can only approximate the overall shape of $Q_{\text {ext }}$. The free parameter $Q_{0}$ describes the $x$-value where the extinction efficiencies peak. The parameter depends on the condensate optical properties, and thus, on the particle composition. It could therefore be used for a first-order characterization of condensates in exoplanet transmission spectra, for example. These transmission spectra depend mostly on the extinction properties and not on the detailed contributions of scattering and absorption. The idea of such an analytical fit is, thus, to offer simple retrievable parameters for transmission spectroscopy that would allow for a basic characterization of the condensates present in the planet's atmosphere to a certain degree. It will not be accurate enough to distinguish between different members of, for example, the olivine or pyroxene family, though.

In the following subsection, we present our fitting procedure. The corresponding fits and associated errors with respect to the actual $Q_{\text {ext }}$ are discussed in Section 5.2.

\subsection{Description of the analytical fit}

We follow the analytical fit of $Q_{\text {ext }}$ as a function of $x$ proposed in Lee et al. (2013) but increase the number of free parameters to three:

$Q_{\mathrm{ext}}=\frac{Q_{1}}{Q_{0} x^{-a}+x^{0.2}}$,

where $Q_{1}$ is a normalization constant, $Q_{0}$ determines the $x$-value where $Q_{\text {ext }}$ peaks, and $a$ is the slope in the small particle limit. It is quite obvious that a simple, analytical equation as this cannot describe all the usually complex behaviour of the extinction efficiencies over a large wavelength range, especially the various specific absorption features of the different condensates. We may, however, hope to use the analytic expression in restricted wavelength bands associated with important observational filters. 

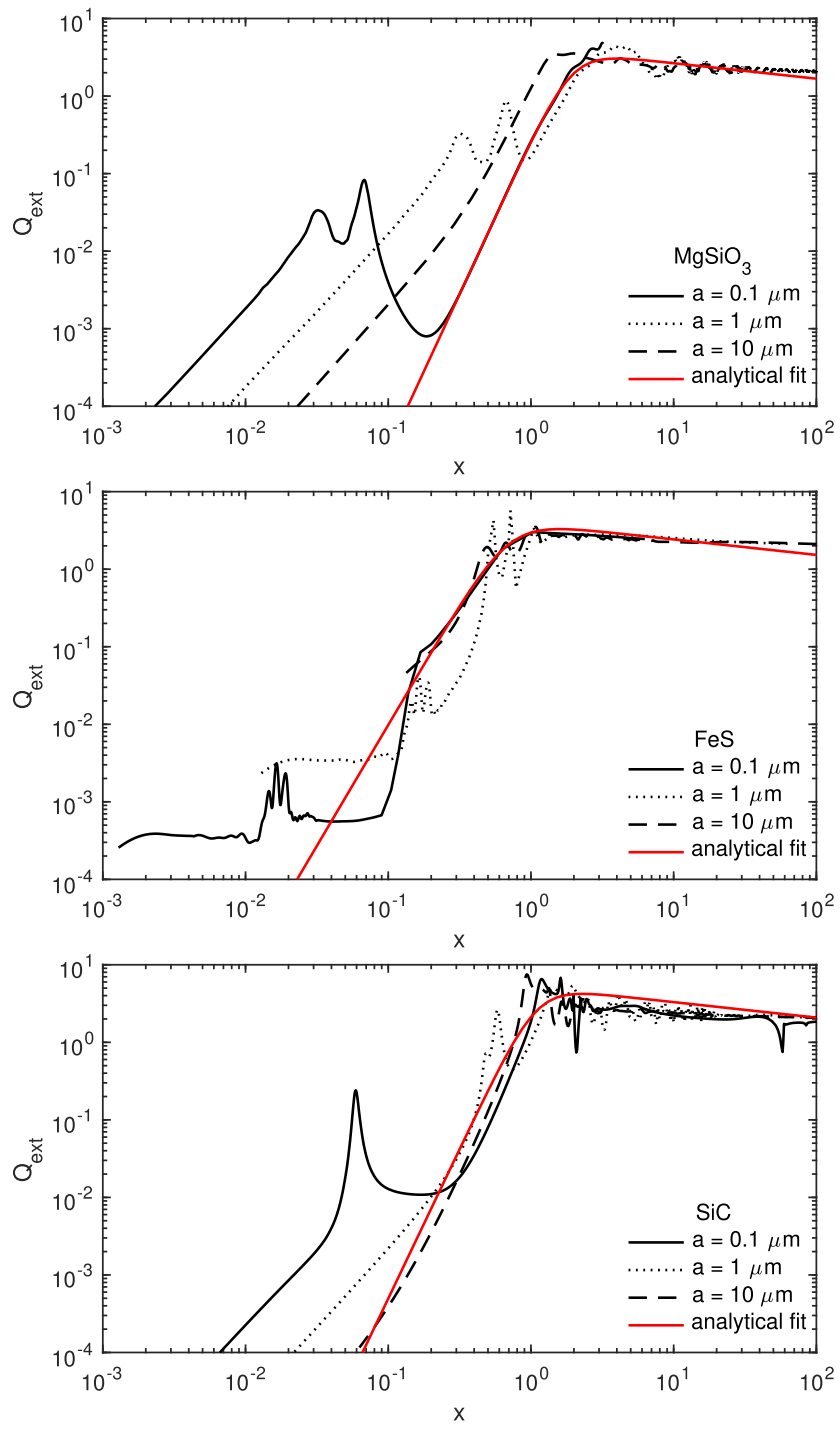

Figure 3. Examples of analytical fits for three different condensates. The analytical fits are depicted by the red, solid curves as a function of the size parameter $x$. For comparison, the $Q_{\text {ext }}$ values are also shown in black for three different particles sizes: $0.1 \mu \mathrm{m}$ (solid), $1 \mu \mathrm{m}$ (dotted), and $10 \mu \mathrm{m}$ (dashed).

Here, we focus especially on the WFC3 instrument with the G141 grism and the standard filters $J, H$, and $K$ that are used in exoplanet transmission or brown dwarf emission spectroscopy. In principle, one could make an independent fit for each filter separately. For simplicity, we use WFC3 here in the following. To constrain the three parameters, we perform a global minimization by using a threedimensional Nelder-Mead simplex method (Lagarias et al. 1998). The quantity to be minimized is the mean error in the WFC3 filter, integrated over a range of particle sizes with radii between $a_{l}=0.1 \mu \mathrm{m}$ and $a_{u}=10 \mu \mathrm{m}$ :

$\bar{E}=\frac{1}{\log a_{u}-\log a_{l}} \int_{\log a_{l}}^{\log a_{u}} E(a) \mathrm{d} \log a$,

with the mean error $E$ in a wavelength range between $\lambda_{l}$ and $\lambda_{u}$ given by

$E(a)=\frac{1}{\lambda_{u}-\lambda_{l}} \int_{\lambda_{l}}^{\lambda_{u}}\left|Q_{\text {ext,mie }}(a, \lambda)-Q_{\text {ext,fit }}(a, \lambda)\right| \mathrm{d} \lambda$.
Table 2. Coefficients of the analytic fits of the extinction efficiencies and their corresponding average errors. The errors shown in the table refer to the WFC3 filter The condensates are ordered with respect to their $Q_{0}$ value.

\begin{tabular}{|c|c|c|c|c|}
\hline Condensate & $Q_{0}$ & $Q_{1}$ & $a$ & $\bar{E}$ (per cent) \\
\hline $\mathrm{C}$ & 0.07 & 3.62 & 6.58 & 8.95 \\
\hline $\mathrm{TiC}$ & 0.24 & 3.60 & 3.74 & 7.45 \\
\hline $\mathrm{Cr}$ & 0.30 & 3.61 & 3.66 & 7.96 \\
\hline $\mathrm{Fe}[\mathrm{s}]$ & 0.30 & 3.59 & 3.88 & 8.09 \\
\hline $\mathrm{FeS}$ & 0.30 & 3.84 & 3.12 & 6.01 \\
\hline $\mathrm{FeO}$ & 0.49 & 3.78 & 4.31 & 7.78 \\
\hline $\mathrm{Fe}_{2} \mathrm{O}_{3}$ & 0.99 & 3.81 & 5.05 & 12.38 \\
\hline $\mathrm{MnS}$ & 1.11 & 3.84 & 5.44 & 15.35 \\
\hline $\mathrm{SiC}$ & 1.47 & 3.86 & 5.23 & 13.19 \\
\hline $\mathrm{TiO}_{2}$ & 1.74 & 3.90 & 5.21 & 13.30 \\
\hline $\mathrm{ZnS}$ & 2.16 & 3.88 & 4.96 & 12.80 \\
\hline $\mathrm{CaTiO}_{3}$ & 2.17 & 3.94 & 5.07 & 12.88 \\
\hline $\mathrm{SiO}$ & 2.48 & 4.02 & 4.25 & 8.66 \\
\hline $\mathrm{Mg}_{0.8} \mathrm{Fe}_{1.2} \mathrm{SiO}_{4}$ & 3.56 & 4.00 & 3.55 & 10.05 \\
\hline $\mathrm{MgFeSiO}_{4}$ & 4.68 & 3.99 & 3.53 & 10.45 \\
\hline $\mathrm{Fe}_{2} \mathrm{SiO}_{4}$ & 5.52 & 4.09 & 4.38 & 11.28 \\
\hline $\mathrm{Na}_{2} \mathrm{~S}$ & 6.77 & 4.07 & 4.03 & 10.79 \\
\hline $\mathrm{MgO}$ & 8.23 & 4.09 & 4.15 & 10.18 \\
\hline $\mathrm{Mg}_{0.4} \mathrm{Fe}_{0.6} \mathrm{SiO}_{3}$ & 8.51 & 4.09 & 3.92 & 9.93 \\
\hline $\mathrm{MgAl}_{2} \mathrm{O}_{4}$ & 8.85 & 4.10 & 4.18 & 9.90 \\
\hline $\mathrm{Mg}_{0.5} \mathrm{Fe}_{0.5} \mathrm{SiO}_{3}$ & 9.54 & 4.10 & 3.96 & 9.57 \\
\hline $\mathrm{Al}_{2} \mathrm{O}_{3}$ & 9.55 & 4.16 & 3.51 & 9.99 \\
\hline Titan tholins & 10.69 & 4.15 & 4.07 & 9.49 \\
\hline $\mathrm{Mg}_{2} \mathrm{SiO}_{4}$ & 11.95 & 4.16 & 4.05 & 9.29 \\
\hline $\mathrm{Mg}_{0.8} \mathrm{Fe}_{0.2} \mathrm{SiO}_{3}$ & 13.27 & 4.18 & 3.95 & 8.66 \\
\hline $\mathrm{MgSiO}_{3}{ }^{a}$ & 15.15 & 4.17 & 3.98 & 8.93 \\
\hline $\mathrm{MgSiO}_{3}{ }^{b}$ & 15.59 & 4.21 & 3.98 & 8.80 \\
\hline $\mathrm{SiO}_{2}{ }^{c}$ & 16.47 & 4.21 & 3.95 & 8.79 \\
\hline $\mathrm{NaCl}$ & 16.59 & 4.22 & 3.95 & 8.74 \\
\hline $\mathrm{KCl}$ & 20.76 & 4.18 & 3.84 & 8.68 \\
\hline $\mathrm{H}_{2} \mathrm{O}[\mathrm{s}]$ & 64.98 & 4.36 & 3.34 & 9.53 \\
\hline
\end{tabular}

Notes. ${ }^{a}$ amorphous (glassy); ${ }^{b}$ amorphous (sol-gel);

${ }^{c} \alpha$-crystal $\&$ amorphous.

The integration over the particle sizes in the calculation of $\bar{E}$ is performed over the logarithm of $a$ because the radii span two orders of magnitude. We limit the largest radii to $a_{u}=10 \mu \mathrm{m}$ because larger particles would already be in the large particle limit at the small wavelengths under investigation here, i.e. their extinction efficiencies would yield $Q_{\text {ext }}=2$, irrespective of the actual particle size. The efficiencies have been calculated with the Mie code described in Section 2 for a broad range different particle radii between $a_{u}=0.1 \mu \mathrm{m}$ and $a_{u}=10 \mu \mathrm{m}$. Examples of the resulting extinction efficiencies for three different sizes and three different condensates are shown in Fig. 3.

\subsection{Fit results}

The obtained fit coefficients and the mean error $\bar{E}$ are shown in Table 2 in descending order of their $Q_{0}$ parameter. As mentioned in Lee et al. (2013), refractory condensates feature $Q_{0}$ values around 10-20, while more volatile species, such as water, have much higher ones. The $Q_{0}$ values of elements like iron, chromium, or graphite, on the other hand, drop below unity. Based on this range of values, it might be possible to identify condensates present in exoplanet atmosphere to a certain degree by employing retrieval techniques (see e.g. Lavie et al. 2017). 


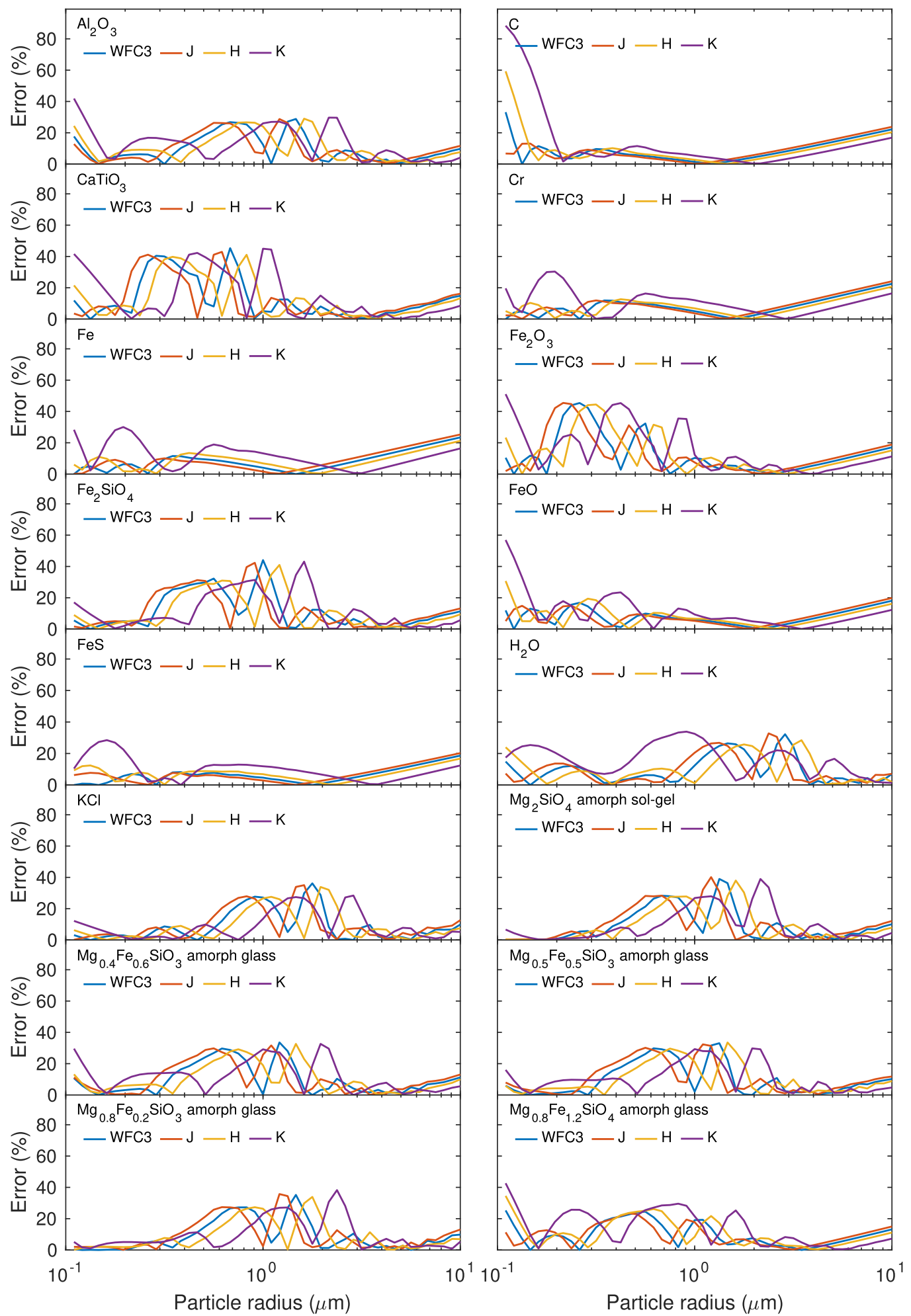

Figure 4. Errors of the analytical fits as a function of particle radius. The errors are shown for four different filters commonly used in exoplanet and brown dwarf spectroscopy: G141@WFC3 (blue), $J$ (red), $H$ (yellow), and $K$ (purple).

The normalization constant $Q_{1}$ shows a much smaller spread, with values close to 4 . The exponential parameter varies roughly between three and seven, depending on the absorptivity of the materials in the wavelength range under consideration. Thus, for actual retrieval methods, the normalization constant $Q_{1}$ could be fixed to four in many cases since the accuracy of exoplanet data is currently not good enough to distinguish the very close parameter values for many of the condensates.

Examples of the analytical fits in comparison to the actual $Q_{\text {ext }}$ curves obtained from Mie theory are shown in Fig. 3 for three 


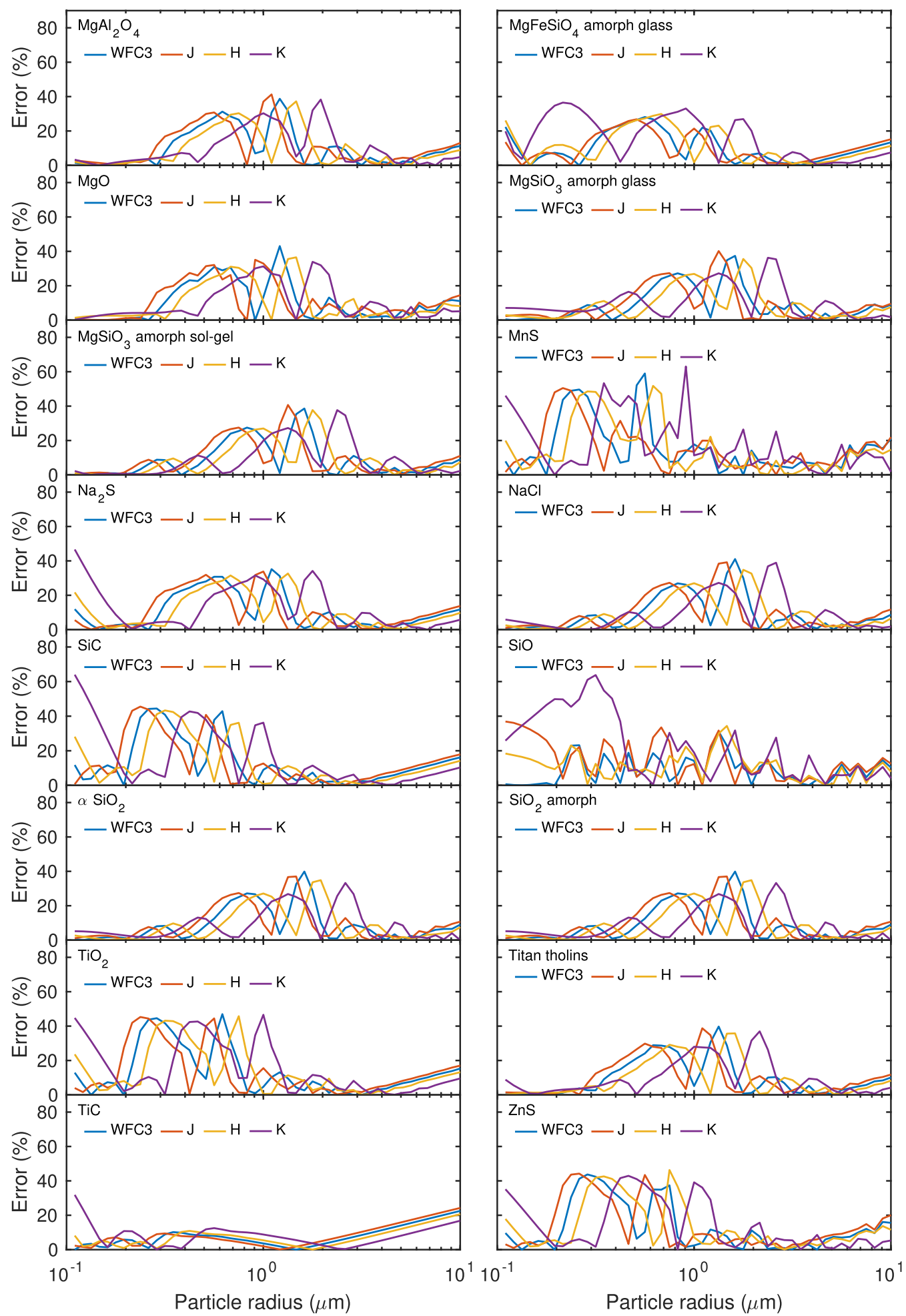

Figure 4 - continued

different condensates. The results suggest that the analytical function can represent the actual Mie efficiencies in certain wavelength ranges. Obviously, the simple fit cannot account for absorption features of the different condensates, as can be seen for the cases of FeS or moissanite.
More detailed information on the errors of the fits as a function of particle sizes are shown in Fig. 4. The figure depicts the mean errors, averaged over four different filters, as a function of the particle sizes. Equation (34) is used to calculate the mean error in each filter. The results shown in Fig. 4 suggest that depending on the 
type of condensate and the particle size, the errors can be as small as 1 per cent, but can also yield values around 80 per cent, especially for very small particle radii.

\section{DISCUSSION AND PROSPECTS FOR FUTURE WORK}

In this work, we compiled a new set of optical constants for potential condensates in atmospheres of giant exoplanets and brown dwarfs. While we tried to provide a consistent data set over a broad wavelength range for each species, the data needs to be treated with care as the lack of available laboratory data currently does not allow us to provide more accurate refractive indices or for intercomparisons since in most cases only single measurements were available. Gaps in the experimental data needed to be closed by extrapolation of the $n$ or $k$ values and by employing the KramersKronig relations. Other uncertainties for exoplanet atmospheres include also the effect of temperature or porosity on the optical constants.

Optical constants are clearly a function of temperature (see e.g. Zeidler et al. 2013). Temperature-dependent effects include, for example, a shift and/or broadening of absorption features or changes in the general slopes of the $n$ and $k$ values. In most cases, however, the refractive index has been measured at room temperature or in environments resembling the cold interstellar medium. Data for temperatures more related to the atmospheres of brown dwarfs or extrasolar giant planets are rarely available, and measurements for these cases are strongly required. Additionally, the optical constants of sulphide-bearing species need additional laboratory measurements to close the gaps in the present data, especially for $\mathrm{MnS}$ and $\mathrm{FeS}$. Furthermore, most solid condensates forming in atmospheres are expected to be amorphous, which also directly impacts the refractive index. To date, unfortunately, this data is unavailable for many condensates, though the measurements by, for example, Dorschner et al. (1995) or Jäger et al. (2003) are notable exceptions. The compilation of optical constants listed in Table 1 and shown in Fig. 1 is available as part of this publication's online material and on the Exoclime Simulation Platform (http://www.exoclime.org).

The optical properties of the condensates were calculated by using our newly developed, improved Mie-scattering code. It is able to calculate the Mie efficiencies, asymmetry parameters, and scattering phase functions for size parameters exceeding values of $10^{7}$. The code has been extensively tested against other available codes. It will be publicly released as part of the Exoclime Simulation Platform. With the calculated optical properties, we performed analytical fits of the obtained extinction efficiencies. The fits can be used in atmospheric retrieval of exoplanet atmospheres by adding two to three additional parameters to the set of retrievable quantities that describe the basic shapes of the extinction efficiencies. They, thus, allow for a basic characterization of the clouds present in these atmospheres. We will employ these analytical fits for atmospheric retrieval in a future publication.

\section{ACKNOWLEDGEMENTS}

The authors knowledge financial and administrative support by the Center for Space and Habitability and the PlanetS National Centre of Competence in Research (NCCR), as well as funding from the Swiss National Science Foundation (SNSF). DK would like to thank $\mathrm{ABC}$ Patzer for helping to obtain the optical constants of TiC.

\section{REFERENCES}

Abramowitz M., Stegun I. A., 1972, Handbook of Mathematical Functions. Dover Press, New York

Arney G. et al., 2016, Astrobiology, 16, 873

Begemann B., Dorschner J., Henning T., Mutschke H., Gürtler J., Kömpe C., Nass R., 1997, ApJ, 476, 199

Birnstiel T., Klahr H., Ercolano B., 2012, A\&A, 539, A148

Bohren C. F., Huffman D. R., 1998, Absorption and Scattering of Light by Small Particles. WILEY-VCH, Weinheim

Cachorro V., Salcedo L., 1991, J. Electromagn. Waves Appl., 5, 913

Chandrasekhar S., 1960, Radiative Transfer. Dover Press, New York

Colaprete A., Toon O. B., 2003, J. Geophys. Res. (Planets), 108, 5025

Dave J. V., 1970, Appl. Opt., 9, 1888

Dorschner J., Begemann B., Henning T., Jaeger C., Mutschke H., 1995, A\&A, 300, 503

Draine B. T., 2003, ApJ, 598, 1017

Du H., 2004, Appl. Opt., 43, 1951

Fabian D., Henning T., Jäger C., Mutschke H., Dorschner J., Wehrhan O., 2001, A\&A, 378, 228

Helling C., Woitke P., Thi W.-F., 2008, A\&A, 485, 547

Heng K., 2016, ApJ, 826, L16

Henning T., Mutschke H., 1997, A\&A, 327, 743

Henning T., Mutschke H., 2001, Spectrochim. Acta, 57, 815

Henning T., Begemann B., Mutschke H., Dorschner J., 1995, A\&AS, 112, 143

Huffman D. R., Wild R. L., 1967, Phys. Rev., 156, 989

Infeld L., 1947, Quart. Appl. Math., 5, 113

Jäger C., Dorschner J., Mutschke H., Posch T., Henning T., 2003, A\&A, 408, 193

Kangarloo H., Rafizadeh S., 2010a, 2nd International Conference on Mechanical and Electronics Engineering. IEEE, New York, p. V2-136

Kangarloo H., Rafizadeh S., 2010b, 2nd International Conference on Mechanical and Electronics Engineering. IEEE, New York, p. V2-237

Khachai H., Khenata R., Bouhemadou A., Haddou A., Reshak A. H., Amrani B., Rached D., Soudini B., 2009, J. Phys.: Condens. Matter., 21, 095404

Khare B. N., Sagan C., Arakawa E. T., Suits F., Callcott T. A., Williams M. W., 1984, Icarus, 60, 127

Kitzmann D., Patzer A. B. C., von Paris P., Godolt M., Stracke B., Gebauer S., Grenfell J. L., Rauer H., 2010, A\&A, 511, A66

Koide T., Shidara T., Fukutani H., Fujimori A., Miyahara T., Kato H., Otani S., Ishizawa Y., 1990, Phys. Rev. B, 42, 4979

Koike C., Kaito C., Yamamoto T., Shibai H., Kimura S., Suto H., 1995, Icarus, 114, 203

Kramers H. A., 1927, Atti Cong. Intern. Fisica, 2, 545

Kronig R. D. L., 1926, J. Opt. Soc. Am., 12, 547

Lagarias J. C., Reeds J. A., Wright M. H., Wright P. E., 1998, SIAM J. Optim., 9, 112

Laor A., Draine B. T., 1993, ApJ, 402, 441

Lavie B. et al., 2017, AJ, 154, 91

Lee J.-M., Heng K., Irwin P. G. J., 2013, ApJ, 778, 97

Lentz W. J., 1976, Appl. Opt., 15, 668

Lucarini V., Peiponen K.-E., Saarinen J. J., Vartiainen E. M., 2005, KramersKronig Relations in Optical Materials Research. Springer-Verlag, Heidelberg

Marley M. S., Ackerman A. S., Cuzzi J. N., Kitzmann D., 2013, Clouds and Hazes in Exoplanet Atmospheres. University of Arizona Press, Tucson, AZ, p. 367

Mie G., 1908, Ann. Phys., Lpz., 330, 377

Montaner A., Galtier M., Benoit C., Bill H., 1979, Physica Status Solidi Appl. Res., 52, 597

Mordasini C., 2014, A\&A, 572, A118

Morley C. V., Fortney J. J., Marley M. S., Visscher C., Saumon D., Leggett S. K., 2012, ApJ, 756, 172

Oreshenko M. et al., 2017, ApJ, 847, L3

Palik E. D., 1985, Handbook of Optical Constants of Solids. Academic Press, Cambridge 
Palik E. D., 1991, Handbook of Optical Constants of Solids II. Academic Press, Cambridge

Pierrehumbert R. T., 2010, Principles of Planetary Climate. Cambridge Univ. Press, Cambridge

Pinhas A., Madhusudhan N., 2017, MNRAS, 471, 4355

Pollack J. B., Hollenbach D., Beckwith S., Simonelli D. P., Roush T., Fong W., 1994, ApJ, 421, 615

Pont F., Knutson H., Gilliland R. L., Moutou C., Charbonneau D., 2008, MNRAS, 385, 109

Posch T., Kerschbaum F., Fabian D., Mutschke H., Dorschner J., Tamanai A., Henning T., 2003, ApJS, 149, 437

Rakić A. D., Djurišić A. B., Elazar J. M., Majewski M. L., 1998, Appl. Opt., 37,5271

Rother T., 2009, Electromagnetic Wave Scattering on Nonspherical Particles. Springer-Verlag, Heidelberg

Schmitt C. G., Heymsfield A. J., Connolly P., Järvinen E., Schnaiter M., 2016, Geophys. Res. Lett., 43, 11

Shen J., Cai X., 2005, in Progress in Electromagnetics Research Symposium. EWW Publishing, Cambridge, p. 691

Siefke T. et al., 2016, Adv. Opt. Mater., 4, 1780

Sing D. K. et al., 2016, Nature, 529, 59

Stevenson K. B., Bean J. L., Seifahrt A., Gilbert G. J., Line M. R., Désert J.-M., Fortney J. J., 2016, ApJ, 817, 141

Tsiaras A. et al., 2017, ApJ, preprint (arXiv:1704.05413)

Ueda K., Yanagi H., Noshiro R., Hosono H., Kawazoe H., 1998, J. Phys.: Condens. Matter., 10, 3669

van de Hulst H. C., 1957, Light Scattering by Small Particles. John Wiley $\&$ Sons, New York

Wakeford H. R., Sing D. K., 2015, A\&A, 573, A122

Warren S. G., 1984, Appl. Opt., 23, 1206

Wiscombe W. J., 1979, Technical report. National Center for Atmospheric Research, Boulder

Wiscombe W. J., 1980, Appl. Opt., 19, 1505

Zeidler S., Posch T., Mutschke H., Richter H., Wehrhan O., 2011, A\&A, 526, A68

Zeidler S., Posch T., Mutschke H., 2013, A\&A, 553, A81

\section{SUPPORTING INFORMATION}

Supplementary data are available at MNRAS online.

Supplementary data are available at (Collection of optical constants for a set of potential condensates in atmospheres of extrasolar planets and brown dwarfs.)

Please note: Oxford University Press is not responsible for the content or functionality of any supporting materials supplied by the authors. Any queries (other than missing material) should be directed to the corresponding author for the article.

\section{APPENDIX A: MIE SCATTERING CODE VALIDATION}

The Mie code developed in this work has been extensively validated against the results of other standard Mie codes, such as the wellknown code MIEvo (Wiscombe 1979). In this appendix, we present a sample of the validation results.

For the intercomparison, we use a set of refractive indices published by e.g. Du (2004) or Shen \& Cai (2005), which includes cases of strongly, weakly, and non-absorbing particles. The values

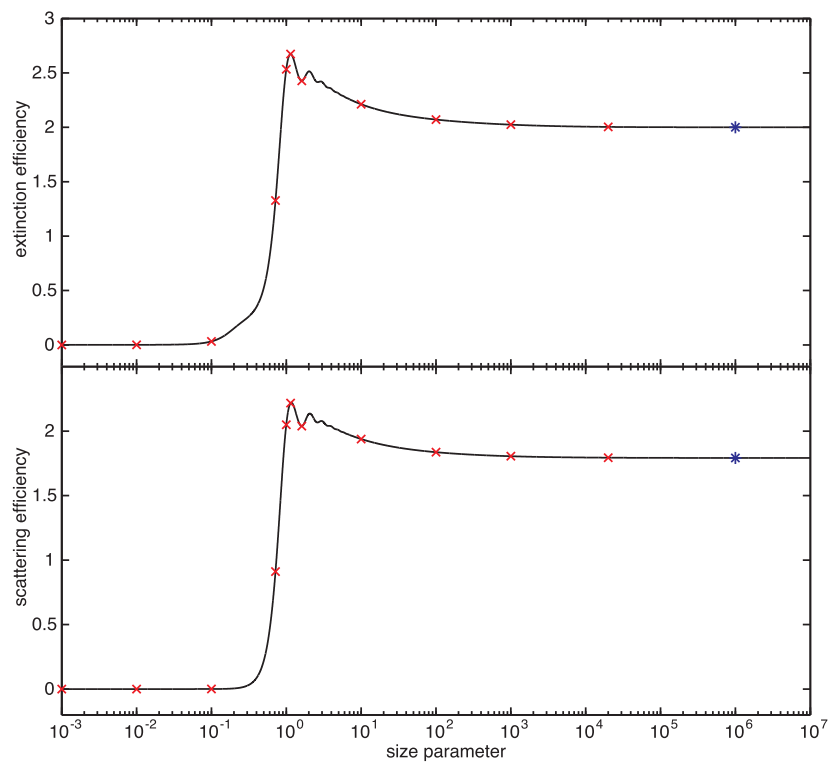

Figure A1. Extinction efficiency (upper diagram) and scattering efficiency (lower diagram) obtained from Mie scattering calculations with LX-MIE for a refractive index of $m=10-10$ i. Selected results of the MIEvo code from Wiscombe (1979) are shown as red crosses for several selected representative size parameters. Additionally, the results for $x=10^{6}$ published by Du (2004) are marked by blue stars.

for the extinction efficiency $Q_{\text {ext }}$ and scattering efficiency $Q_{\text {sca }}$ obtained from our code LX-MIE, MIEVo (in double-precision accuracy), and published results from Du (2004) are listed in Table A1 for different refractive indices $m$ and several specific size parameters $x$. Additionally, the extinction and scattering efficiencies for a refractive index of $m=10-10 \mathrm{i}$ are shown in Fig. A1 as a function of the size parameter $x$. Selected values for representative size parameters calculated with MIEVO are also shown in this figure within its validated range of $x<20000$. For a quantitative comparison, some of these values are listed in Table A1.

The resulting efficiencies in Table A1 and Fig. A1 show that the results of our Mie code and MIEVo agree in at least nine significant digits in most cases. Some minor deviations are found for small size parameters, which are caused by the small-particle approximations used in MIEVO (see Wiscombe 1979, for details on its treatment of Mie calculations for small size parameters). Note, that the results from $\mathrm{Du}$ (2004) listed in Table A1 are identical to those published by Shen \& Cai (2005), who introduced the improved treatment of the Riccati-Bessel functions, which is now also used in LX-MIE.

For a large size parameter of $x=10^{6}$, the values given in $\mathrm{Du}$ (2004) are used for comparison. His results of $Q_{\text {ext }}=2.00022$ and $Q_{\text {sca }}=1.79218$ agree with our calculated values in all digits. While $x=10^{6}$ is the largest size parameter for which results were published by $\mathrm{Du}$ (2004), Fig. A1 suggests that LX-MIE yields numerically stable results for size parameters of at least $10^{7}$. Thus, LX-MIE provides accurate results over at least 10 orders of magnitude in the size parameter range. 
Table A1. Comparison of extinction and scattering efficiencies obtained from Du (2004), MIEVo (Wiscombe 1979), and LX-MIE (this work) for different refractive indices and size parameters.

\begin{tabular}{|c|c|c|c|c|c|c|c|}
\hline \multirow[b]{2}{*}{$m$} & \multirow[b]{2}{*}{$x$} & \multicolumn{3}{|c|}{$Q_{\mathrm{ext}}$} & \multicolumn{3}{|c|}{$Q_{\text {sca }}$} \\
\hline & & Du (2004) & MIEVO & LX-MIE & Du (2004) & MIEVO & LX-MIE \\
\hline $10-10 \mathrm{i}$ & 0.001 & & $6.00207512 \times 10^{-5}$ & $6.00207581 \times 10^{-5}$ & & $2.66646989 \times 10^{-12}$ & $2.66646989 \times 10^{-12}$ \\
\hline $10-10 \mathrm{i}$ & 0.1 & & $3.20390865 \times 10^{-2}$ & $3.20390907 \times 10^{-2}$ & & $2.70983004 \times 10^{-4}$ & $2.70983004 \times 10^{-4}$ \\
\hline $10-10 \mathrm{i}$ & 1 & 2.53299 & 2.53299308 & 2.53299308 & 2.04941 & 2.04940501 & 2.04940501 \\
\hline $10-10 \mathrm{i}$ & 100 & 2.07112 & 2.07112433 & 2.07112433 & 1.83679 & 1.83678540 & 1.83678540 \\
\hline $10-10 \mathrm{i}$ & 1000 & & 2.02426046 & 2.02426046 & & 1.80546582 & 1.80546582 \\
\hline $10-10 \mathrm{i}$ & 20000 & & 2.00361474 & 2.00361474 & & 1.79419080 & 1.79419080 \\
\hline $10-10 \mathrm{i}$ & $10^{6}$ & 2.00022 & & 2.00021914 & 1.79218 & & 1.79218105 \\
\hline $1.5-1 \mathrm{i}$ & 100 & 2.09750 & 2.09750176 & 2.09750176 & 1.28370 & 1.28369705 & 1.28369705 \\
\hline $1.5-1 \mathrm{i}$ & 10000 & 2.00437 & 2.00436771 & 2.00436771 & 1.23657 & 1.23657431 & 1.23657431 \\
\hline $1.33-10^{-5} \mathrm{i}$ & 100 & 2.10132 & 2.10132071 & 2.10132071 & 2.09659 & 2.09659351 & 2.09659351 \\
\hline $1.33-10^{-5} \mathrm{i}$ & 10000 & 2.00409 & 2.00408893 & 2.00408893 & 1.72386 & 1.72385722 & 1.72385722 \\
\hline $0.75-0 \mathrm{i}$ & 10 & 2.23226 & 2.23226484 & 2.23226484 & 2.23226 & 2.23226484 & 2.23226484 \\
\hline $0.75-0 \mathrm{i}$ & 1000 & 1.99791 & 1.99790818 & 1.99790818 & 1.99791 & 1.99790818 & 1.99790818 \\
\hline $0.75-0 \mathrm{i}$ & 10000 & & 2.00125518 & 2.00125518 & & 2.00125518 & 2.00125518 \\
\hline
\end{tabular}

This paper has been typeset from a $\mathrm{TE}_{\mathrm{E}} \mathrm{L} / \mathrm{LT} \mathrm{E} \mathrm{X}$ file prepared by the author. 